TIFR/TH/95-05

DO-TH-95-03

IISC-CTS- $1 / 95$

May 25, 1995

hep-ph/9507341

\title{
Bloch-Nordsieck Thermometers: One-loop Exponentiation in Finite Temperature QED.
}

\author{
Sourendu Guptaf, D. Indumathif, P. Mathews?, V. Ravindran'
}

\begin{abstract}
We study the scattering of hard external particles in a heat bath in a real-time formalism for finite temperature QED. We investigate the distribution of the 4-momentum difference of initial and final hard particles in a fully covariant manner when the scale of the process, $Q$, is much larger than the temperature, $T$. Our computations are valid for all $T$ subject to this constraint. We exponentiate the leading infrared term at one-loop order through a resummation of soft (thermal) photon emissions and absorptions. For $T>0$, we find that tensor structures arise which are not present at $T=0$. These carry thermal signatures. As a result, external particles can serve as thermometers introduced into the heat bath. We investigate the phase space origin of $\log (Q / m)$ and $\log (Q / T)$ terms.
\end{abstract}

\footnotetext{
${ }^{1}$ Theory Group, Tata Institute of Fundamental Research, Homi Bhabha Road, Bombay 400005, India, Email—sgupta@theory.tifr.res.in

${ }^{2}$ Lehrstuhl für Theoretische Physik IV, D-44221, Dortmund, Germany, Emailindu@hal1.physik.uni-dortmund.de

${ }^{3}$ Centre for Theoretical Studies, Indian Institute of Science, Bangalore 560012, India, Email_prakash@cts.iisc.ernet.in

${ }^{4}$ Theory Group, Tata Institute of Fundamental Research, Homi Bhabha Road, Bombay 400005, India, Email— ravi@theory.tifr.res.in
} 


\section{Introduction}

Recently there has been a lot of phenomenological interest in external particles interacting inside a heat-bath. This is due to the realisation that hard jets formed in the early stages of a heavy-ion collision can interact with a quark-gluon plasma formed later, and perhaps carry away information on this plasma [1, 2]. However, such processes are often infra-red sensitive. Phenomenological studies have used arbitrary infra-red cutoffs to obtain finite results. It is legitimate to ask whether one can remove this arbitrariness and obtain stable finite results for such infra-red sensitive processes.

In this paper we consider the simpler, but related, problem of hard scatterings of some external particles introduced within a heat-bath at a temperature $T$. We choose two of the simplest processes in QED - the scattering of an electron with an off-shell photon and the annihilation of an electronpositron pair into an off-shell photon. Due to quantum and thermal fluctuations, extra soft particles can also be involved in each of these scatterings.

If $p^{\text {in }}$ and $p^{\text {out }}$ are the summed momenta of all the hard initial and final state particles respectively, then one can construct the difference

$$
P_{\mu}=p_{\mu}^{\text {in }}-p_{\mu}^{\text {out }}
$$

We study the distribution of $P$ through the measurement of a suitable semiinclusive cross section. It is non-trivial because of the extra soft particles. When $P^{2} \ll Q^{2}$ ( $Q^{2}$ is the scale of the hard process), the Lorentz-invariant cross section factorises as

$$
\frac{d^{4} \sigma}{d P^{4}} \approx \sigma_{0} \frac{d^{4} \mathcal{P}}{d P^{4}}
$$

where $\sigma_{0}$ is the inclusive cross section for the process. Throughout this paper we assume such a factorisation, and study the distribution of $P_{\mu}$ (the second factor on the right hand side) through its cumulants, i.e., the connected parts of the moments.

Naively, it might seem that the heat-bath spoils the Lorentz invariance of various cross sections. This is incorrect. The bath only selects a frame; Lorentz invariance cannot be lost due to the existence of a special frame. Cross sections which are Lorentz invariant at $T=0$ remain so for $T>0$. We

carry out a fully covariant treatment of the resummation and show that the difference between $T=0$ and $T>0$ physics lies in some new asymmetries and 
angular correlations. These are proportional to powers of the temperature. As a result, scatterings of external particles can be used as thermometers for the heat-bath. This is the main result of this paper.

It is useful to recall the status of infra-red sensitive cross sections at $T=0$. Exclusive scattering cross sections can be infra-red singular. The analysis due to Bloch and Nordsieck [3] showed that such measurements are impossible to make with any real detector. They argued that a real detector, with a finite energy resolution, measures, at best, some semi-inclusive cross section. They computed one such cross section and showed that it is indeed non-singular. In the limit where the detector becomes ideal, i.e., the resolution becomes infinitely sharp, this cross section remains non-singular.

A stronger theorem is also available for $T=0$ field theories; it goes by the name of the Kinoshita-Lee-Nauenberg theorem [4]. The theorem states that inclusive cross sections are infra-red finite, order by order in perturbation theory. Infra-red singularities in real and virtual diagrams cancel in each order. Semi-inclusive cross sections are also infra-red finite. However, incomplete cancellations between real and virtual parts show up as large logarithms. It is possible to sum these to all orders in perturbation theory.

At finite temperatures the infra-red singularities could be stronger, since every squared matrix element comes with a thermal distribution function attached to each external leg. Each external boson then adds one power to the infra-red divergence. A $T>0$ version of the KLN theorem has not been proved. Computations have been performed in several models to check whether or not infra-red divergences cancel in suitably defined inclusive or semi-inclusive processes [5, 6].

An important recent result is due to Landshoff and Taylor [7]. In a leading order perturbative computation of the decay width for a scalar going to two scalars, they found exact cancellation of the infra-red singularity. Weldon introduced a crucial simplification in finite temperature QED [8]. By eikonalising the soft part of the one-loop order cross section, he was able to resum soft photon effects to all orders in perturbation theory and obtain the distribution $d \mathcal{P} / d P_{0}$. This is an extension of the Bloch-Nordsieck theorem to finite temperature.

This proof is a crucial input to our work. We extend it first to the case of interest to us, i.e., the full momentum resummation. We construct a Lorentz invariant characteristic function for the distribution of $P$, by exponentiating the first order perturbative correction to the hard process. Then 
we extract the cumulants of this distribution and find terms of the form $\log (Q / m)$. These large logarithms come from parts of phase space where the extra photons are nearly collinear with the fermion momenta, exactly as at $T=0$. These large terms allow us to identify phenomenologically interesting results from such soft photon resummations at finite temperature. We also find terms in $\log (Q / T)$. These arise from terms left over after cancellation of the infra-red divergence.

The plan of this paper is the following. In Section 2 we shall perform the resummation and show that Weldon's proof of infra-red finiteness 8 . can be generalised in a Lorentz invariant way to yield the distribution of $P$. An outline of a diagrammatic proof of the cancellation of infra-red divergences is given in Appendix A. In Section o 3 we examine the mean and covariance of $P_{\mu}$ at $T=0$, since this is the base against which the $T>0$ results have to be compared. In Section 1 the corresponding $T>0$ quantities are computed. Various details of these calculations are relegated to Appendices B-D. Our results and their implications are discussed in Section 5. The reader who is not interested in the details of the computation may skip to this section.

\section{The Resummed Distribution}

In this paper we assume that a factorisation of the hard and soft parts of the cross section holds in the form given in eq. (1.2), and then examine the soft part of the cross section, i.e., the distribution of $P_{\mu}$. The generating function for all matrix elements needed for a resummation of the soft part of the one-loop order contribution is [8],

$$
Z(J, T)=\int \mathcal{D} A_{\mu} \exp \left[-i \int d^{4} x\left(\frac{1}{4} F_{\mu \nu} F^{\mu \nu}+J_{\mu} A^{\mu}\right)\right] .
$$

The integral over $x_{0}$ in the exponent is carried out over a path in the complex time plane. The choice of this path is made so that the thermal averaging of the S-matrix elements is done properly. Detailed discussions of the path of integration can be found in [9]. Some more information can be found in Appendix A.

At this point it is useful to introduce some notation. For the scattering process, the initial and final momenta of the electron are $p$ and $p^{\prime}$, and the initial photon has spacelike 4-momentum $q\left(q^{2}=-Q^{2}\right)$. For the annihilation process, the electron momentum is $p$, the positron momentum is $p^{\prime}$ and 
the final photon momentum $q^{\prime}$ is timelike $\left(q^{\prime 2}=Q^{2}\right)$. Details of the hard scattering kinematics are given in Appendix B.

A proof of the factorisation implicit in eq. (1.2) can be obtained by considering the leading perturbative corrections to the hard scattering process and taking the limit of the extra photon momentum, $k$, going to zero (see Appendix A). For the processes we consider, this gives

$$
J_{\mu}(k)=i e\left(\frac{p_{\mu}^{\prime}}{p^{\prime} \cdot k}-\frac{p_{\mu}}{p \cdot k}\right) .
$$

Here $e$ is the positron charge. It is important to remember that this approximation is valid only in the infra-red. Hence, in order to use eqs. (2.1) and (2.2) consistently, we must truncate the mode expansion of the soft fields in the partition function.

We perform this truncation in a Lorentz invariant fashion by writing the mode expansion-

$$
\begin{aligned}
A_{\mu}(x) & =\sum_{\lambda} \int \tilde{d} k\left[\epsilon_{\mu}^{*}(k, \lambda) a_{\lambda}^{\dagger}(k) \mathrm{e}^{i k \cdot x}+\epsilon_{\mu}(k, \lambda) a_{\lambda}(k) \mathrm{e}^{-i k \cdot x}\right], \\
\tilde{d k} & =\frac{d^{4} k}{(2 \pi)^{4}} 2 \pi \delta\left(k^{2}\right) \Theta\left(k_{0}\right) \Theta\left(\eta V^{2}-V \cdot k\right) .
\end{aligned}
$$

Here $\epsilon_{\mu}(k, \lambda)$ is the polarisation vector for a photon of momentum $k$ and polarisation $\lambda$, and $a_{\lambda}(k)$ and $a_{\lambda}^{\dagger}(k)$ are the annihilation and creation operators. $V$ is a vector built out of the momenta of the hard particles which sets the scale of the hard process and $\eta \ll 1$. The second theta-function in the Lorentz invariant phase space measure expresses the fact that the fields are soft.

Note that such a factorised approach is useful only when there is a great difference between the hard and soft scales. In particular, we take all thermal fluctuations into the soft scale, and hence we must have $Q^{2} \gg T^{2}$. For both the processes, we choose $V$ to be the timelike vector $q^{\prime}\left(q^{\prime}=p+p^{\prime}\right.$ in the hard-scattering kinematics). The arguments in this section do not depend on the choice of $V$.

In the Feynman gauge, the generating function involves only Gaussian integrals. Performing them we obtain

$$
Z(J, T)=\exp \left(-\frac{i}{2} \int \frac{d^{4} k}{(2 \pi)^{4}} J^{\mu}(k) G_{\mu \nu}(k, u) J^{\nu}(-k)\right)
$$


where the thermal photon propagator is given by

$$
G_{\mu \nu}(k, u)=-g_{\mu \nu}\left\{\frac{1}{k^{2}+i \epsilon}-2 \pi i \delta\left(k^{2}\right) B(k \cdot u ; T)\right\},
$$

and the Bose distribution function is

$$
B(k \cdot u ; T)=\frac{1}{\mathrm{e}^{|k \cdot u| / T}-1} .
$$

The Lorentz vector $u\left(u^{2}=1\right)$ specifies the 4 -velocity of the heat bath with respect to the observer [10]. In the rest frame of the heat bath, $k \cdot u=k_{0}$, and the Bose distribution takes its familiar form. This is a covariant way of expressing the fact that the presence of the heat bath selects out a preferred frame.

The matrix element for $n$-photon emission is obtained by taking appropriate derivatives of $Z(J, T)$ with respect to the external current, giving

$$
\mathcal{M}\left(k_{1}, \cdots, k_{n}\right)=Z(J, T) \prod_{i=1}^{n} \epsilon_{\mu}\left(k_{i}\right) J^{\mu}\left(k_{i}\right) .
$$

Emission of photons corresponds to taking $k_{i}^{0}>0$, and absorption to $k_{i}^{0}<0$. The squared matrix element, after summation over polarisations, gives a factor of $J^{2}$ for each photon. Finite temperature effects in the cross-section may be completely taken into account through an extension of the usual trick (see Appendix A) of writing a phase space element

$$
d \phi_{i}=\frac{d^{4} k_{i}}{(2 \pi)^{4}} 2 \pi \delta\left(k_{i}^{2}\right)\left[\Theta\left(k_{i}^{0}\right)+B\left(k_{i} \cdot u ; T\right)\right] \Theta\left(\eta q^{\prime 2}-q^{\prime} \cdot k_{i}\right) .
$$

The resummation now gives the probability density for radiation as

$$
\frac{d \mathcal{P}}{d^{4} P}=|Z(J, T)|^{2} \sum_{n=0}^{\infty} \frac{1}{n !} \int \prod_{i=0}^{n}\left\{d \phi_{i} J_{\mu}\left(k_{i}\right) J^{\mu}\left(k_{i}\right)\right\} \delta^{4}\left(P^{\mu}-\sum_{i=0}^{n} k_{i}^{\mu}\right) .
$$

For further analysis we construct the Lorentz invariant characteristic function of this distribution-

$$
\Xi(x) \equiv \int d^{4} P \mathrm{e}^{i P \cdot x} \frac{d \mathcal{P}}{d^{4} P}=\exp \left[R_{0}(x)+R_{T}(x)\right] .
$$


The Fourier transformation decouples the delta function, allowing an exponentiation in the form shown.

The logarithm of the characteristic function is defined by the expressions

$$
\begin{aligned}
& R_{0}=\int \tilde{d} k J(k) \cdot J(k)\left\{\mathrm{e}^{i k \cdot x}-1\right\}, \\
& R_{T}=\int \tilde{d k} J(k) \cdot J(k) B(k \cdot u ; T)\left\{\mathrm{e}^{i k \cdot x}+\mathrm{e}^{-i k \cdot x}-2\right\} .
\end{aligned}
$$

The subtractions come from $|Z(J, T)|^{2}$ and may be obtained from eq (2.4). All infrared singularities cancel between the exponential terms and the constant piece in both the $T=0$ part, $R_{0}$ and the $T>0$ contribution, $R_{T}$. This is an extension of Weldon's proof of infrared finiteness in this model [8].

The characteristic function, $\Xi$, is a generating function for the moments of $P_{\mu}$. The logarithm, $R_{0}+R_{T}$, is a generating function for the "connected parts" of these moments, i.e., the cumulants. The $n$-th cumulant of $P_{\mu}$ is given by

$$
\left\langle P_{\mu_{1}} P_{\mu_{2}} \cdots P_{\mu_{n}}\right\rangle_{c}=\left.(-i)^{n} \frac{\partial}{\partial x_{\mu_{1}}} \frac{\partial}{\partial x_{\mu_{2}}} \cdots \frac{\partial}{\partial x_{\mu_{n}}}\left(R_{0}+R_{T}\right)\right|_{x=0},
$$

where the subscript $c$ denotes a connected part. Each cumulant of $P$ then becomes the sum of a $T=0$ and a $T>0$ part.

Since the integral representation of $R_{T}$ is even in $x$, odd derivatives vanish. As a result, there is no $T>0$ contribution to the odd cumulants. The physics behind this is simple. At finite temperature the charged particle can remove momentum from the heat bath by absorbing a photon, as well as add momentum by emitting. In thermal equilibrium these two processes balance out, giving a distribution even in $P$. It is possible to pursue this further. The symmetry under $x \leftrightarrow-x$ comes from the phase space in eq. (2.8), in particular from the $\Theta+B$ factor there. This is obtained by summing the weights for absorption, $B\left(k_{0}\right) \Theta\left(-k_{0}\right)$, and emission, $\left[1+B\left(k_{0}\right)\right] \Theta\left(k_{0}\right)$. These two weights are related through the commutation relations of the photon field operators. In the ultimate analysis, this is the origin of the vanishing of odd cumulants in thermal equilibrium.

It is useful to note the following result. Combining eqs. (2.11) and (2.12), we find that a cumulant of the resummed distribution is equal to the corresponding moment of the distribution obtained from the soft part of the leading order in perturbation theory. 
We write down explicitly the first cumulant, i.e., the mean radiated momentum-

$$
\left\langle P_{\mu}\right\rangle=-\left.i \frac{\partial}{\partial x_{\mu}}\left(R_{0}+R_{T}\right)\right|_{x=0} .
$$

$\left\langle P_{\mu}\right\rangle$ is given entirely by the $T=0$ piece,

$$
\left\langle P_{\mu}\right\rangle=\int \tilde{d k} J(k) \cdot J(k) k_{\mu}
$$

This is a Lorentz vector. We discuss it in detail later.

We also write down the second cumulant $\left\langle P_{\mu} P_{\nu}\right\rangle_{c}=\left\langle P_{\mu} P_{\nu}\right\rangle-\left\langle P_{\mu}\right\rangle\left\langle P_{\nu}\right\rangle$. This is the covariance of the radiated 4-momentum-

$$
\begin{aligned}
\left\langle P_{\mu} P_{\nu}\right\rangle_{c} & =-\left.\frac{\partial}{\partial x_{\mu}} \frac{\partial}{\partial x_{\nu}}\left(R_{0}+R_{T}\right)\right|_{x=0}=V_{\mu \nu}^{0}+V_{\mu \nu}^{T}, \quad \text { where } \\
V_{\mu \nu}^{0} & =\int \tilde{d k} J(k) \cdot J(k) k_{\mu} k_{\nu}, \\
V_{\mu \nu}^{T} & =\int \tilde{d k} J(k) \cdot J(k) 2 B(k \cdot u ; T) k_{\mu} k_{\nu} .
\end{aligned}
$$

The covariance is a symmetric Lorentz tensor of rank 2. The diagonal components of this tensor give the variance of the corresponding component of the radiated momentum. The off-diagonal elements specify the correlation between different momentum components. In particular, the space-space components quantify angular correlations in the radiated momentum.

\section{Zero Temperature}

The mean radiated momentum after resummation can be written in terms of all the vectors available in the problem, multiplied by appropriate coefficient functions (see Appendix D). As a result-

$$
\left\langle P_{\mu}\right\rangle=\int \tilde{d k} J_{\lambda}(k) J^{\lambda}(k) k_{\mu}=F_{1}\left(\frac{p_{\mu}}{m}\right)+F_{2}\left(\frac{p_{\mu}^{\prime}}{m}\right) .
$$

It is important to note that the frame dependence of all the tensors is taken entirely into the basis tensors. As a result, the coefficient functions are Lorentz invariant. One can obtain these and the tensor components quite trivially by contracting $\left\langle P_{\mu}\right\rangle$ with each of the vectors on the right hand side. 
For convenience, these Lorentz invariant contractions are evaluated in the Breit Frame (BF), i.e., the frame in which $p$ and $p^{\prime}$ are equal and oppositely directed,

$$
\begin{aligned}
& I_{1} \equiv \frac{p_{\mu}}{m}\left\langle P_{\mu}\right\rangle=\frac{\alpha}{\pi} \eta Q\left[(2 \Psi-1) \Omega_{1}-\Omega_{6}\right], \\
& I_{2} \equiv \frac{p_{\mu}^{\prime}}{m}\left\langle P_{\mu}\right\rangle=\frac{\alpha}{\pi} \eta Q\left[(2 \Psi-1) \Omega_{1}-\Omega_{6}\right] .
\end{aligned}
$$

Here $\alpha=e^{2} / 4 \pi$ and $\Psi$ is defined in eq. (B.1) and the integrals $\Omega_{i}$ in eq. (C.7). The final expressions are obtained in the $\mathrm{BF}$ and we have used the fact that $\Omega_{i}=\Omega_{i}^{\prime}$ in this frame (see Table 1 in Appendix D). The equality of the two contractions translates into the equality of $F_{1}$ and $F_{2}$. In the hard-scattering limit $(Q \rightarrow \infty)$, we find

$$
F_{1}=F_{2}=\frac{\alpha}{\pi} \frac{\eta Q}{\bar{\gamma}}\left[2 \log \left(\frac{Q}{m}\right)-1\right],
$$

where $\bar{\gamma}$ is defined in eq. (B.4). The logarithm of $Q / m$ arises from that part of the phase space in which emitted or absorbed photons are collinear with the electron momenta.

The components of the mean radiated momentum can now be easily evaluated in any frame. In the $\mathrm{BF}$, the only non-zero component is

$$
\left\langle P_{0}\right\rangle=2 \bar{\gamma} F_{1} .
$$

In an arbitrary frame, other non-zero components exist. The BF is a good frame in which to compare the $T=0$ and $T>0$ results because of the simplicity of the vacuum results in this frame.

The variance of the radiated momentum has the tensor decomposition

$$
V_{\mu \nu}^{0}=\int \tilde{d k} J_{\lambda}(k) J^{\lambda}(k) k_{\mu} k_{\nu}=\sum_{i=1}^{4} F_{i}^{0} \mathcal{Z}_{\mu \nu}^{i},
$$

where the four Lorentz tensors are listed in eq. (D.3). The coefficient functions and tensor components can be computed as explained in Appendix D.

We need to write the four contractions

$$
\begin{aligned}
I_{1}^{0} & =\mathcal{Z}_{\mu \nu}^{1} V_{\mu \nu}^{0}=0, \\
I_{2}^{0} & =\mathcal{Z}_{\mu \nu}^{2} V_{\mu \nu}^{0}=\frac{\alpha}{2 \pi} \eta^{2} Q^{2}\left[2 \Psi \Omega_{4}-\Omega_{0}-\Omega_{5}\right], \\
I_{3}^{0} & =\mathcal{Z}_{\mu \nu}^{3} V_{\mu \nu}^{0}=\frac{\alpha}{2 \pi} \eta^{2} Q^{2}\left[2 \Psi \Omega_{4}-\Omega_{0}-\Omega_{5}\right], \\
I_{4}^{0} & =\mathcal{Z}_{\mu \nu}^{4} V_{\mu \nu}^{0}=\frac{\alpha}{2 \pi} \eta^{2} Q^{2}\left[2 \Omega_{0}-\frac{2}{\Psi} \Omega_{4}\right] .
\end{aligned}
$$


The final expression for each contraction is written in the BF. The coefficient functions are obtained by solving a linear system of equations, as explained in Appendix D.

Since the matrix $\mathbf{Z}\left(\mathbf{Z}_{i j}=\mathcal{Z}_{\mu \nu}^{i} \mathcal{Z}_{\mu \nu}^{j}\right.$, see eq. D.4) is symmetric under interchange of $\mathcal{Z}^{2}$ and $\mathcal{Z}^{3}$, and $I_{2}^{0}=I_{3}^{0}$, one must have $F_{2}^{0}=F_{3}^{0}$. In the hard scattering limit, we find

$$
\begin{aligned}
& F_{1}^{0}=-\frac{\alpha}{\pi} \eta Q^{2}, \\
& F_{2}^{0}=F_{3}^{0}=\frac{\alpha}{\pi} \eta Q^{2} \bar{\gamma}^{-2}\left[\log \left(\frac{Q}{m}\right)-1\right], \\
& F_{4}^{0}=\frac{4 \alpha}{\pi} \eta Q^{2} .
\end{aligned}
$$

Note that $F_{2}=F_{3}$, as expected, and that collinear singularities appear in these two coefficient functions.

The tensor components of the covariance can now be written down. We find that in the BF only the diagonal components survive.

$$
\begin{aligned}
V_{00}^{0} & =\frac{\alpha}{\pi} \eta Q^{2}\left[2 \log \left(\frac{Q}{m}\right)-1\right] \\
V_{11}^{0} & =V_{22}^{0}=\frac{\alpha}{\pi} \eta Q^{2}, \\
V_{33}^{0} & =\frac{\alpha}{\pi} \eta Q^{2}\left[2 \log \left(\frac{Q}{m}\right)-3\right] .
\end{aligned}
$$

In a general frame, of course, there will be other components of the covariance. The coefficient functions, eq. (3.7), however, are Lorentz invariant, and may be extracted from measurements in any frame.

Other important details to notice about these expressions are that there are no off-diagonal elements, i.e., there are no angular correlations. Also, the transverse asymmetry $V_{11}^{0}-V_{22}^{0}$ vanishes, and neither of the transverse components of the variance involves any large logarithms. Finally, the logarithms in the other two components of the variance are equal, so that the longitudinal asymmetry $V_{00}^{0}-V_{33}^{0}$ is free from these terms.

\section{Finite Temperature}

The finite temperature piece of the resummed distribution is even in $P$. As a result, the $T>0$ contribution to all odd cumulants vanish. In this section 
we consider only the first non-vanishing cumulant, i.e., the covariance. It has the tensor decomposition

$$
V_{\mu \nu}^{T}=\int \tilde{d k} J_{\lambda}(k) J^{\lambda}(k) 2 B(k \cdot u ; T) k_{\mu} k_{\nu}=\sum_{i=1}^{7} F_{i}^{T} \mathcal{T}_{\mu \nu}^{i},
$$

where our choice of the seven Lorentz tensors is listed in eq. (D.5). Recall that at $T=0$ only four tensors could be constructed. The three extra tensors are due to the extra vector $u$ in the problem. The coefficient functions and tensor components can be computed as before.

We begin by evaluating the contractions $I_{i}^{T}=\mathcal{T}_{\mu \nu}^{i} V_{\mu \nu}^{T}$. These integrals are most conveniently performed in the rest frame of the heat bath ( $\mathrm{HBF})$. As discussed in Appendix C, when $Q \gg T$ the contractions are given to accuracy of order $\exp (-Q / T)$ by

$$
\begin{array}{cc}
I_{1}^{T}= & -I_{5}^{T}=\frac{\pi \alpha}{3} T^{2}\left[\Omega_{2}+\Omega_{2}^{\prime}-2 \Psi \Omega_{3}\right], \\
I_{2}^{T}= & \frac{\pi \alpha}{3} T^{2}\left[2 \gamma\left(\Omega_{1}+\Omega_{6}^{\prime}-2 \Psi \Omega_{1}^{\prime}\right)-\left(\Omega_{0}+\Omega_{5}^{\prime}-2 \Psi \Omega_{4}^{\prime}\right)\right]+\gamma^{2} I_{5}^{T}, \\
I_{3}^{T}= & \frac{\pi \alpha}{3} T^{2}\left[2 \gamma^{\prime}\left(\Omega_{1}^{\prime}+\Omega_{6}-2 \Psi \Omega_{1}\right)-\left(\Omega_{0}+\Omega_{5}-2 \Psi \Omega_{4}\right)\right]+\gamma^{\prime 2} I_{5}^{T}, \\
I_{4}^{T}= & \frac{\pi \alpha}{3} T^{2}\left[2 \Omega_{0}+\left(\frac{\gamma^{\prime}}{\Psi}-2 \gamma\right) \Omega_{1}+\left(\frac{\gamma}{\Psi}-2 \gamma^{\prime}\right) \Omega_{1}^{\prime}\right. \\
& \left.-\frac{1}{\Psi}\left(\Omega_{4}+\Omega_{4}^{\prime}-\gamma \Omega_{6}-\gamma^{\prime} \Omega_{6}^{\prime}\right)\right]+\frac{\gamma \gamma^{\prime}}{\Psi} I_{5}^{T}, \\
I_{6}^{T}= & \frac{\pi \alpha}{3} T^{2}\left[2 \Psi \Omega_{1}^{\prime}-\Omega_{1}-\Omega_{6}^{\prime}\right]-I_{5}^{T}, \\
I_{7}^{T}= & \frac{\pi \alpha}{3} T^{2} \frac{1}{\gamma^{\prime}}\left[2 \Psi \Omega_{1}-\Omega_{1}^{\prime}-\Omega_{6}\right]-I_{5}^{T} .
\end{array}
$$

We have used the approximations given in eqs. (C.8) and (C.9). Note that the two pairs $I_{2}^{T}$ and $I_{3}^{T}$, and $I_{6}^{T}$ and $I_{7}^{T}$ are related by interchange of primed and unprimed quantities. The angular integrals are listed in Table 1 .

We write down only the leading behaviour (largest power of $\bar{\gamma}$ ) of the 
coefficient functions in the hard scattering limit-

$$
\begin{aligned}
F_{1}^{T}= & -\frac{\pi \alpha T^{2}}{3} \frac{2 \log (1+A)}{A}, \\
F_{2}^{T}= & \frac{\pi \alpha T^{2}}{3} \frac{1}{\bar{\gamma}^{2}} \frac{1}{\gamma_{-}^{2} A^{2}}\left[A(1-2 A)+2 A^{2} \log (2 \bar{\gamma})\right. \\
& \left.-\left(1+2 A-A^{2}\right) \log \gamma_{-}-(1+A)^{2} \log \gamma_{+}\right], \\
F_{3}^{T}= & \frac{\pi \alpha T^{2}}{3} \frac{1}{\bar{\gamma}^{2}} \frac{1}{\gamma_{+}^{2} A^{2}}\left[A(1-2 A)+2 A^{2} \log (2 \bar{\gamma})\right. \\
& \left.\quad-(1+A)^{2} \log \gamma_{-}-\left(1+2 A-A^{2}\right) \log \gamma_{+}\right], \\
F_{4}^{T}= & \frac{\pi \alpha T^{2}}{3} \frac{4[A-\log (1+A)]}{A^{2}}, \\
F_{5}^{T}= & \frac{\pi \alpha T^{2}}{3}[4 \log (2 \bar{\gamma})-2], \\
F_{6}^{T}= & \frac{\pi \alpha T^{2}}{3} \frac{2}{A}\left[2 A \log (2 \bar{\gamma})-A-(1-A) \log \gamma_{-}-(1+A) \log \gamma_{+}\right], \\
F_{7}^{T}= & \frac{\pi \alpha T^{2}}{3} \frac{2}{A}\left[2 A \log (2 \bar{\gamma})-A-(1+A) \log \gamma_{-}-(1-A) \log \gamma_{+}\right] .
\end{aligned}
$$

In order to display these results in a compact form, we have introduced the notation

$$
x=\beta_{u} \cos \chi, \quad \gamma_{ \pm}=\gamma_{u}(1 \pm x), \quad A=\gamma_{+} \gamma_{-}-1=\gamma_{u}^{2} \beta_{u}^{2} \sin ^{2} \chi,
$$

where $\beta_{u}, \gamma_{u}$ and $\chi$ are defined in eq. (B.4). Since we are interested in the hard-scattering limit, we have only kept the leading power of $\bar{\gamma}$ in these expressions. Note the appearance of $\log (2 \bar{\gamma})=\log (Q / m)$ in several of the coefficient functions.

Although the integrals in eq. (4.2) were performed in the HBF, the coefficient functions are invariant. However, the components of the covariance tensor are frame dependent. For ease of comparison with the $T=0$ results 
in eq. (3.8), we write the non-vanishing components in the BF-

$$
\begin{aligned}
V_{00}^{T}= & \left(\frac{\pi \alpha T^{2}}{3}\right) \frac{2}{(1+A)\left(1-x^{2}\right)}\left[\left(1+x^{2}\right) \log \left(\frac{Q^{2}}{m^{2}}\right)+\left(2 A-1-3 x^{2}\right)\right. \\
& \left.+(1+x)^{2} \log \gamma_{-}+(1-x)^{2} \log \gamma_{+}\right], \\
V_{11}^{T}= & \left(\frac{\pi \alpha T^{2}}{3}\right) 2\left[2-\frac{1}{A} \log (1+A)\right], \\
V_{22}^{T}= & \left(\frac{\pi \alpha T^{2}}{3}\right) \frac{2}{A} \log (1+A), \\
V_{33}^{T}= & \left(\frac{\pi \alpha T^{2}}{3}\right) \frac{2}{(1+A)\left(1-x^{2}\right)}\left[\left(1+x^{2}\right) \log \left(\frac{Q^{2}}{m^{2}}\right)+\left(2 A x^{2}-x^{2}-3\right)\right. \\
V_{01}^{T}= & \left(\frac{\pi \alpha T^{2}}{3}\right) \frac{4}{\left(1-x^{2}\right)} \beta_{u} \sin \chi, \\
V_{03}^{T}= & \left(\frac{\pi \alpha T^{2}}{3}\right) \frac{2}{(1+A)\left(1-x^{2}\right)}\left[2 x \log \left(\frac{Q^{2}}{m^{2}}\right)+2 x(A-2)+\right. \\
V_{13}^{T}= & \left(\frac{\pi \alpha T^{2}}{3}\right) \frac{4}{\left(1-x^{2}\right)} x \beta_{u} \sin \chi .
\end{aligned}
$$

A check is provided by the fact that the diagonal elements of this tensor are positive.

We find several interesting features in eq. (4.5). Most obviously, there are some non-zero off-diagonal components of the covariance tensor. Recall that $p, p^{\prime}$ and $u$ together define the reaction plane. Then all in-plane components of $P$ are correlated with each other, but not with the off-plane component. Furthermore the transverse components of $P$ no longer have equal variance. This is also due to the distinction between in-plane and off-plane components. Finally, all the logarithmically divergent terms arise only in the energy and longitudinal parts of the tensor. Exactly as at $T=0$, the coefficients of the logarithms in the diagonal components are equal.

An invariant comparison of the vacuum $(T=0)$ and $T>0$ results can be made using the coefficient functions. For this we need to convert the tensor expansion in eq. (4.1) into an expansion in terms of the set $S_{\mu \nu}^{i}$ (eq. D.7), since the first four tensors in this set are precisely equal to the tensor set used at $T=0$. The coefficient functions in this basis are $\tilde{F}_{i}^{T}=\sum_{j} F_{j}^{T} \mathbf{S}^{i j}$. Using the explicit form for $\mathbf{S}$ given in eq. (D.8), we see that $\tilde{F}_{i}^{T}=F_{i}^{T}$ for $i=1, \cdots, 4$. A 

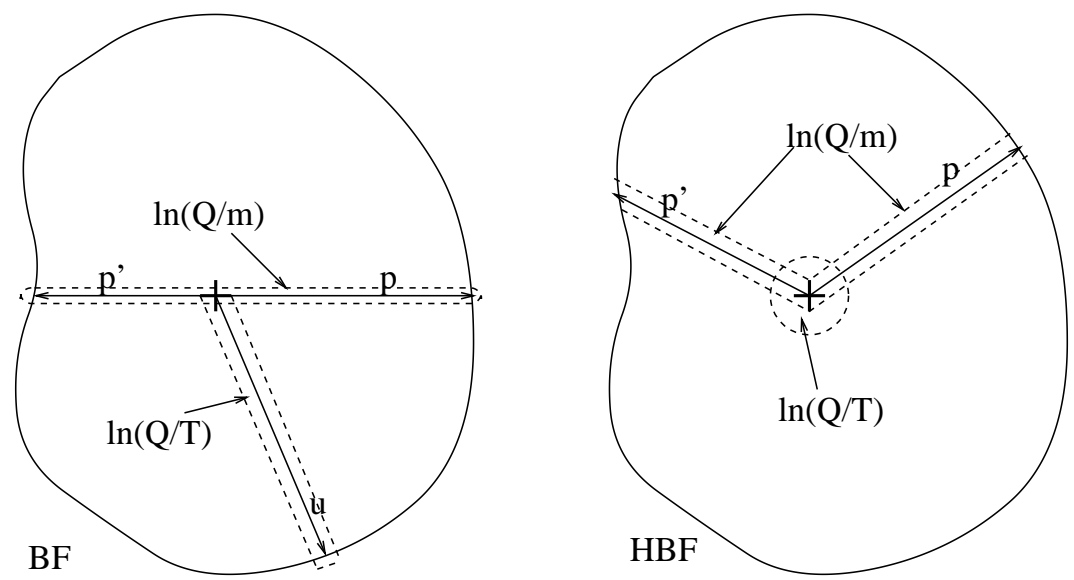

Figure 1: The regions of the photon phase space from which one obtains different logarithmic terms are included within dashed lines. The directions of the vectors in the problem are shown. Note that in the HBF the origin (marked with a cross) is not included in the integration because of infra-red cancellations. In the BF the ray parallel to $u$ is removed.

comparison of eq. (3.7) with the first four expressions in eq. (4.3) shows the change due to the temperature. Apart from the differences in the coefficients of the $\log (Q / m)$ terms, there are new $\log \gamma_{ \pm}$terms. Using the arguments in Appendix $\mathrm{Q}$ (see the discussion following eq. C.9) it is easy to see that these are terms in $\log (Q / T)$.

The origin of the logarithms is shown in Figure 1. The $\log (Q / m)$ terms arise from the same source at $T=0$ and $T>0$. They come from the part of phase space where the photon momentum $k$ is nearly parallel to $p$ or $p^{\prime}$. The $\log (Q / T)$ terms, on the other hand, arise entirely from the thermal distribution. This is clear from eq. (4.1), since the $\gamma_{u}$ dependence of the results can come only from the $k \cdot u$ term when the integrals are performed in the BF. Hence, these logarithms can be identified as due to the part of the phase space where $k \cdot u \ll T$. Since this is a Lorentz invariant 0 , we can transform this to the $\mathrm{HBF}$, where it becomes equivalent to saying that the logarithms arise in the region of phase space where $k_{0} \ll T$.

The $\log (Q / T)$ terms never give large contributions. One way of understanding this is to note that the actual singularity in the Bose distribution is removed through a cancellation between the real and virtual diagrams. 
This is equivalent to removing the origin in the phase space of $k$. Thus, in the limit $T \rightarrow 0$, when the Bose distribution becomes concentrated on the origin, its contribution to the process actually vanishes. In other words, positive powers of $T$ always regulate the divergence in $\log T$. Contrast this to the collinear parts. Along the rays parallel to $p$ or $p^{\prime}$ in phase space there are actual singularities. These regions become available as $m \rightarrow 0$, and hence give rise to genuinely large contributions to various observables.

When $Q>T \gg m$, then the covariances provide straightforward measurements of the temperature. Useful observables are the two asymmetries

$$
A_{L}=\frac{\left\langle P_{0} P_{0}\right\rangle_{c}-\left\langle P_{3} P_{3}\right\rangle_{c}}{\left\langle P_{0} P_{0}\right\rangle_{c}+\left\langle P_{3} P_{3}\right\rangle_{c}}, \quad A_{T}=\frac{\left\langle P_{1} P_{1}\right\rangle_{c}-\left\langle P_{2} P_{2}\right\rangle_{c}}{\left\langle P_{1} P_{1}\right\rangle_{c}+\left\langle P_{2} P_{2}\right\rangle_{c}},
$$

and the correlation functions

$$
C_{\mu \nu}=\frac{\left\langle P_{\mu} P_{\nu}\right\rangle_{c}}{\sqrt{\left\langle P_{\mu} P_{\mu}\right\rangle_{c}+\left\langle P_{\nu} P_{\nu}\right\rangle_{c}}} .
$$

In the BF, $A_{T}, C_{01}, C_{03}$ and $C_{13}$ are precisely zero at $T=0$, but become nonzero at finite temperatures. Each of these is proportional to $(T / \eta Q)^{2}$ and hence need not be very small. As a result, they can yield measurements of the temperature. Furthermore, all other correlations remain zero even at finite temperature. The pattern of vanishing and non-vanishing correlations, or equivalently, off-diagonal components of the covariance tensor shows whether or not any observed effects are due to finite temperatures. As a result, these observations provide not only measurements of the temperature, but also cross checks that the effects do indeed come from thermal effects.

We do not discuss numbers here since such estimates require matching of the perturbative and resummed parts of the cross section [11. It would nevertheless be interesting to carry out such a computation to see whether it is possible to design special purpose experiments to measure these effects.

\section{Discussion and Conclusions}

We have performed a resummation of soft photon emissions in two hard processes in finite temperature QED. These are the scattering of an electron by a hard spacelike photon, and the annihilation of an electron positron pair into a hard timelike photon. 
The summation has been performed in a fully Lorentz-invariant manner. This was achieved by separating soft and hard photons invariantly, eq. (2.3), and by writing the thermal photon propagator invariantly, eq. (2.5). This second step reveals that finite temperature physics involves one extra Lorentz vector over the corresponding vacuum process [10. This is a Lorentzinvariant way of stating that a heat-bath selects out a preferred frame.

We showed that an invariant resummation of soft photon effects is possible and yields a perfectly finite distribution of the total radiated photon momentum, P. Thus Weldon's extension [8] of the Bloch-Nordsieck theorem [3, 12] can itself be extended from energy distributions to the full 4-momentum distribution. The net effect of finite temperatures is to produce correlations in the radiation pattern. By performing a tensor decomposition of the $\mathrm{cu}-$ mulants of $P$, we obtained a Lorentz-invariant comparison of the radiation pattern in vacuum and in a heat-bath in terms of certain scalar coefficient functions, eqs. (3.7) and (4.3). At finite temperature, large logarithms appear in unfamiliar places.

The comparison of these results is simplest in the BF. In this frame there is a rotational symmetry around the common direction of the two fermion momenta at $T=0$. Furthermore, there is a symmetry in the interchange of the two momenta. As a result, the mean momentum vanishes, leaving only a non-zero mean radiated energy, eq. (3.4). Also, there are no correlations between the different components of $P$, i.e., the covariance tensor is diagonal. The transverse diagonal components of this tensor are equal due to the rotational symmetry. Large logarithms show up in the other two diagonal components, eq. (3.8).

At finite temperatures both these symmetries are spoilt due to the presence of the extra vector $u$. The mean of $P$ is not changed, since momentum can be absorbed from the heat bath as well as emitted. For a process in equilibrium, these two rates balance out, as seen from the symmetry of $R_{T}$ (eq. 2.11) under $x \rightarrow-x$. However, the covariance tensor changes substantially (eq. 4.5). The extra vector, $u$, allows an invariant distinction between transverse momentum in the reaction plane and off it. The variances of these two components are no longer related by any symmetry, and hence can be different. All the in-plane components of $P$ are correlated with each other, but not with the off-plane component. As a result, the covariance tensor is no longer diagonal. In particular, the covariance of the energy and longitudinal momenta contains a large logarithm, and may be easily measured. 
We notice that the $T>0$ results contain two types of logarithms - either in $Q / m$ or in $Q / T$. The source of the former is the same in the vacuum and finite temperature theories; they arise from parts of the phase space where the radiated (or absorbed) photon is nearly collinear with the fermion momenta. In the $m \rightarrow 0$ limit photons can become precisely collinear with the fermions. This configuration gives a truly divergent contribution to various observables. As a result, the $\log (Q / m)$ terms can become large. The $\log (Q / T)$ terms arise only at $T>0$, and are due to soft parts of the phase space (in the HBF). The Born-level singularity at $k=0$ is removed by cancellation between real and virtual diagrams, and these logarithms never give large contributions. It appears [6] that for hard scattering of thermal particles the $\log (Q / m)$ terms do not arise. In this sense, the scattering of external particles in a heat bath seems to be closer to the familiar situation at zero temperature.

When including hard thermal loops, the formal considerations of section 2 change in two ways (see Appendix A). First, the phase space element in eq. (2.8) has to be modified, by taking into account the absorptive part of the Braaten-Pisarski resummed photon propagator [13]. Second, the current in eq. (2.2) must be rederived using this resummed photon propagator in the leading perturbative correction. The formal results are otherwise unchanged provided the cancellation of infra-red singularities can be shown.

Consequently, we expect the arguments in the rest of this paper to remain valid after Braaten-Pisarski resummation. In particular, odd cumulants will still vanish in the thermal part of the resummed distribution. There will still be seven coefficient functions for the covariance tensor, and the zero and non-zero components can still be identified by the methods developed here. Detailed computations including hard thermal loops are in progress, and will be reported later.

The extension of these results to one-loop order QCD resummations is straightforward. We refer to the resummation procedure discussed in [14 in the context of gluon radiative corrections to the Drell-Yan process. The phase space measure there is written in the Sudakov parametrisation. Nevertheless, it is easy to check that the resummation of the leading soft singularity in oneloop order corrections gives an exponentiation of $J_{\mu}(k) J^{\mu}(k)$. This current is very similiar to that in eq. (2.2). The only differences are that $m^{2}=0$ in [14 and that factors of $\alpha$ are replaced by $\alpha_{s} C_{F}$. Since no non-Abelian couplings appear in QCD to this order, all of Appendix A can be translated to that context with only these changes. 
The upshot is that the resummation procedure outlined here also generalises to one-loop order resummation in finite temperature QCD. All our formulæ can be carried over by the simple replacement $\alpha \rightarrow \alpha_{s} C_{F}$. We expect that the logarithmic mass singularities can be absorbed into the structure functions of the external hadrons which undergo hard scattering inside the heat-bath.

There are obvious phenomenological consequences of the computations presented here. Each off-diagonal component of the covariance tensor can be measured independently. A separate measurement of the temperature is obtained from each non-zero component. These estimates must agree with each other, and from that obtained by measuring $A_{L}$ and $A_{T}$. A further experimental check is that all off-plane components of this tensor are indeed zero. These conditions being fulfilled would verify that the observations are due to thermal effects, and simultaneously give a measurement of the temperature. In summary, soft photon resummations yield a new thermometer in high energy physics.

Acknowledgements: This work was started during the "Micro-workshop on Hard Probes of the Quark Gluon Plasma", held in TIFR, Bombay, in December 1994. We would like to thank Avijit Ganguly and Rohini Godbole for useful discussions during the workshop. We would also like to thank Rohini Godbole for a careful reading of the manuscript.

\section{A The Resummation Procedure}

Here we give a diagrammatic proof of the resummed result to order $\alpha$ for the processes considered in the text using the techniques of [7]. The hard scattering cross sections with soft thermal photon contributions can be obtained in perturbation theory. The hard particles are external and do not thermalise in the heat bath. The interaction Lagrangian is

$$
\mathcal{L}_{I}=\mathcal{L}_{I}^{h}+\mathcal{L}_{I}^{s}
$$

where $\mathcal{L}_{I}^{h}$ is written in terms of the hard part of the photon field and $\mathcal{L}_{I}^{s}$ in terms of the soft part-

$$
\mathcal{L}_{I}^{h}=j_{\mu}^{h}(x) \cdot A_{h}^{\mu}(x) \quad \text { and } \quad \mathcal{L}_{I}^{s}=j_{\mu}^{h}(x) \cdot A_{s}^{\mu}(x) .
$$


In this appendix we use the notation $j^{\mu} \cdot A_{\mu}=\int d^{4} x j^{\mu}(x) A_{\mu}(x)$ and the current $j_{\mu}=i e \bar{\psi} \gamma_{\mu} \psi$. In order to make the computation transparent, we quantise the fields in a box of volume $\mathrm{V}$ and take infinite volume limit at the end. Thus,

$$
A_{\mu}^{s}(x)=\sum_{l}^{\prime} \frac{1}{\sqrt{2 k_{l} V}}\left[a_{l} \epsilon_{\mu}\left(k_{l}\right) \exp \left(-i k_{l} \cdot x\right)+a_{l}^{\dagger} \epsilon_{\mu}^{*}\left(k_{l}\right) \exp \left(i k_{l} \cdot x\right)\right] .
$$

The prime over the summation denotes that it involves only soft modes. The continuum Lorentz invariant version of this is given in eq. (2.3).

In terms of the transition matrix, $\mathbf{T}$, and the density matrix for the thermal fields, $\rho$, the inclusive cross section is

$$
\begin{aligned}
\sigma & =\frac{1}{F} \operatorname{Tr}\left(\rho \mathbf{T} \mathbf{T}^{\dagger}\right) \\
& =\frac{1}{Z_{0} F} \sum_{\{m\},\{n\}, e^{\prime}} \exp \left(-\sum_{l} \beta n_{l} \epsilon_{l}\right)\left|\left\langle\{m\}, e^{\prime}|\mathbf{T}|\{n\}, e, \gamma^{*}\right\rangle_{c}\right|^{2},
\end{aligned}
$$

where $\quad Z_{0}=\prod_{l}\left[1-\exp \left(-\beta \epsilon_{l}\right)\right]^{-1}$ and $i \mathbf{T}=\exp \left[i \int d x \mathcal{L}_{I}(x)\right]-1$.

The subscript $c$ in the matrix element denotes the connected part. The label $\{n\}$ stands for the set of occupation numbers for each mode of the soft fields, and $F$ is a flux factor. Note, $\rho=\exp (-\beta H) / Z$ where $Z$ is the full partition function of the interacting system. The process of taking connected parts in the second line absorbs the interaction effects and leaves only a normalisation by the ideal gas partition function $Z_{0}$. The density matrix for electrons in the heat bath is disregarded since the hard particles are not thermal averaged and because there are no electron loops to lowest order.

The soft photon contribution to the hard scattering cross section can be obtained from the following part of the transition matrix-

$$
\mathbf{T} \approx T\left[j^{\mu} \cdot A_{\mu}^{h}\left(1+j^{\mu} \cdot A_{\mu}^{s}+\frac{1}{2}\left(j^{\mu} \cdot A_{\mu}^{s}\right)^{2}+\cdots\right)\right]
$$

where $T$ denotes time ordering. This expansion is made keeping the order $\alpha$ term in the hard scattering and all orders in the soft thermal photons. After Wick contractions, this gives two types of graphs - real soft photon emission and absorption, and virtual soft photon corrections. For every field which is thermal averaged the contraction gives a thermal propagator. 

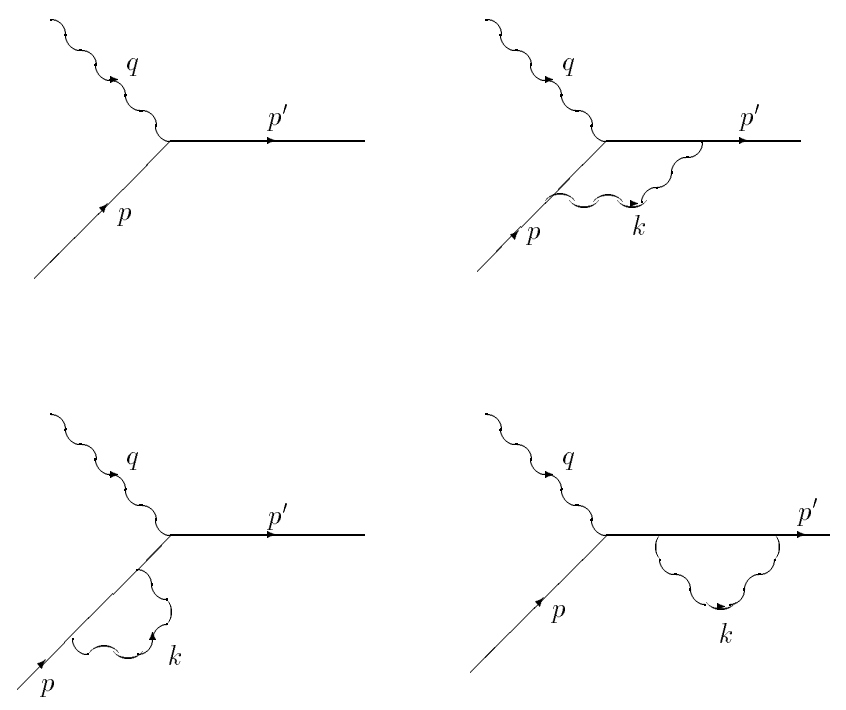

Figure 2: The virtual corrections discussed in eq. (A.7). The first diagram corresponds to the lowest order process in $\sigma_{v}$, and the other three to the parts in $M_{v}$.

To order $\alpha$ the virtual soft photon contribution (Figure 2) to the hard scattering cross section can be obtained from

$$
\mathbf{T}_{v}=\frac{1}{2} T\left(j^{\mu} \cdot A_{\mu}^{h} j^{\alpha} \cdot A_{\alpha}^{s} j^{\beta} \cdot A_{\beta}^{s}\right),
$$

by Wick contracting only the soft thermal photons. To this order there are no thermal fermion loops. Since there are no external soft photons in the virtual part, only the diagonal elements of the transition matrix will contribute. Taking the continuum limit, we obtain the result in an arbitrary frame as

$$
\begin{gathered}
\sigma_{v}=\frac{e^{2}}{F} \int \frac{d^{4} p^{\prime} d^{4} k}{(2 \pi)^{8}} 2 \pi \delta\left(p^{2}-m^{2}\right) \Theta\left(p_{0}^{\prime}\right)(2 \pi)^{4} \delta^{4}\left(p+q-p^{\prime}\right) \\
\left.\times \bar{u}\left(p^{\prime}\right) \phi(q) u(p)\right]^{*}\left[\bar{u}\left(p^{\prime}\right) M_{v} u(p)\right], \\
\text { where } \quad M_{v}=\epsilon_{\mu} \Gamma^{\mu}\left(k, p, p^{\prime}\right)+\Sigma\left(k, p^{\prime}\right) \frac{1}{\not p^{\prime}-m} \phi(q)+\phi(q) \frac{1}{p-m} \Sigma(k, p) .
\end{gathered}
$$

Here $\Sigma(k, p)$ and $\Gamma_{\mu}\left(k, p, p^{\prime}\right)$ are the usual self-energy and vertex functions calculated at $T=0$, only with the photon propagator replaced by the thermal 

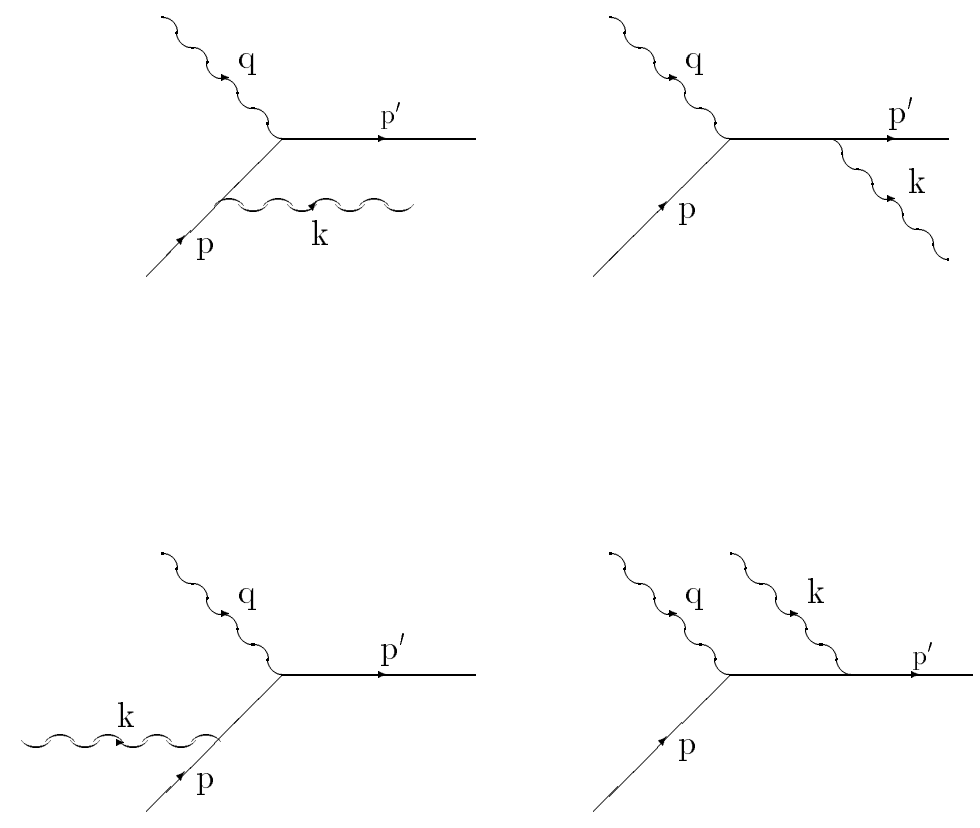

Figure 3: The real photon emission and absorption corrections of $\sigma_{r}$ in eq. (A.10). Absorbed soft photons come in from the left, emitted photons exit to the right. Note that the distinction between emission and absorption is included into the phase space measure.

photon propagator of eq. (2.5). The electron propagators do not have thermal parts, since they are assumed external. In short, this expression is identical to the $T=0$ result but for the thermal photon propagator. This remains true even if one considers the Braaten-Pisarski resummed hot thermal loops [13], although the form of the propagator then changes.

The real soft photon emission and absorption graphs (Figure [3) upto the same order are obtained from the term

$$
\mathbf{T}_{r}=T\left(j^{\mu} \cdot A_{\mu}^{h} j^{\alpha} \cdot A_{\alpha}^{s}\right) .
$$

The matrix element of this operator is proportional to $\left\langle\{m\}\left|A_{\alpha}^{s}\right|\{n\}\right\rangle$. Only the off-diagonal elements of the transition matrix contribute to this cross 
section. Using the identity

$$
\frac{1}{Z_{l}} \sum_{n_{l}} n_{l} \exp \left(-\beta n_{l} k_{l}\right)=B\left(k_{l} ; \beta\right),
$$

(where $\beta=1 / T$ ) we get the following cross section for real photon emission and absorption in the 3 limit-

$$
\begin{aligned}
& \sigma_{r}=\frac{e^{4}}{F} \int \frac{d^{4} p^{\prime}}{(2 \pi)^{4}} 2 \pi \delta\left(p^{\prime 2}-m^{2}\right) \Theta\left(p_{0}^{\prime}\right) d \phi(k) \\
& \times(2 \pi)^{4} \delta^{4}\left(p+q-p^{\prime}-k\right)\left|\bar{u}\left(p^{\prime}\right) M_{r} u(p)\right|^{2}, \\
& \text { where } \quad M_{r}=\phi(q) \frac{1}{\not p^{\prime}+\not k-m} \epsilon^{*}(k)+\epsilon^{*}(k) \frac{1}{\not p-\not k-m} \phi(q) \text {, }
\end{aligned}
$$

and the phase space element for real thermal photons is given by eq. (2.8) This phase space for $T>0$ changes because of the additional absorption processes which do not exist in the vacuum theory. Again, the electron propagators do not have thermal parts. The important point is that in both the real and virtual corrections, the matrix elements are the same as at zero temperature. Finite temperature effects are subsumed into the thermal photon phase space and propagator.

We note that the squared matrix element appearing in $\sigma_{r}$ amounts to taking the one loop corrections to the basic process with a cut on the soft photon line. The phase space thus comes from the absorptive part of the thermal photon propagator. Upon performing Braaten-Pisarski resummation, the real photon phase space element will then be changed in obvious ways.

The soft photon limit is obtained by setting $k$ to zero in the numerator of the matrix elements in $\sigma_{v}$ and $\sigma_{r}$. In this limit we get

$$
\begin{gathered}
{\left[\bar{u}\left(p^{\prime}\right) \xi u(p)\right]^{*} \quad\left[\bar{u}\left(p^{\prime}\right) M_{v} u(p)\right]=-\left|\bar{u}\left(p^{\prime}\right) M_{r} u(p)\right|^{2}} \\
=\left[\epsilon_{\mu}(k) J^{\mu}(k)\right]^{2}\left|\bar{u}\left(p^{\prime}\right) \xi(q) u(p)\right|^{2}
\end{gathered}
$$

where $J^{\mu}$ is the current in eq. (2.2). This is the origin of the factorisation in eq. (1.2). Taking higher order terms from eq. (A.5) gives the exponentiation

\footnotetext{
${ }^{1}$ In section 2 a Lorentz invariant cutoff on the soft modes has also been included with this. The rest of the phase space element was also written down in 8].
} 
of the order $\alpha$ result. It is possible to show that a factorisation in the form shown in eq. (A.11) holds with higher powers of $\left(\epsilon_{\mu} J^{\mu}\right)^{2}$. As a result, we obtain the squares of the matrix elements shown in eq. (2.7), and hence the generating function of eq. (2.1). A full proof will be given elsewhere.

\section{B Hard-Scattering Kinematics}

The problem considered here contains three Lorentz vectors, the two fermion momenta, $p$ and $p^{\prime}$, and the velocity vector of the heat-bath, $u$. With three vectors we can construct three Lorentz invariants. Since $p^{2}=p^{\prime 2}=m^{2}$ and $u^{2}=1$, we can work with the dimensionless invariants

$$
\frac{1}{m} p \cdot u=\gamma, \quad \frac{1}{m} p^{\prime} \cdot u=\gamma^{\prime}, \quad \text { and } \quad \frac{1}{m^{2}} p \cdot p^{\prime}=\Psi .
$$

For $T=0$, the problem does not involve $u$ and the only invariant available is $\Psi$.

For the electron scattering problem, it is a convention to use the square of the vector $q=p^{\prime}-p$ as one of the invariants. This is a measure of the scale of the process. We have the relation

$$
Q^{2} \equiv-q^{2}=2 m^{2}(\Psi-1)
$$

The limit of a hard scattering, $Q^{2} \rightarrow \infty$, corresponds to taking $\Psi \rightarrow \infty$. The momentum $q^{\prime}=p+p^{\prime}$ is closely related. In the annihilation process this corresponds to the final photon momentum, and its square to its invariant mass. We have,

$$
q^{\prime 2}=2 m^{2}(\Psi+1) \stackrel{\Psi \rightarrow \infty}{\longrightarrow} Q^{2} .
$$

Note that $q$ is space-like and $q^{\prime}$ time-like, and $q \cdot q^{\prime}=0$. We choose to use $q^{\prime}$ to set the scale of the hard scattering process.

We shall find it most convenient to work in one of two frames, either the rest frame of the heat-bath $(\mathrm{HBF})$ or the Breit frame $(\mathrm{BF})$. In this latter frame, $p$ and $p^{\prime}$ have equal and opposite 3-momenta, and their common direction conventionally defines the $z$-direction. For the annihilation process the $\mathrm{BF}$ is just the rest frame of the final off-shell photon. The reaction plane in the HBF is chosen to be the $x z$-plane. Then this is also the plane containing $p, p^{\prime}$ and $u$ in the $\mathrm{BF}$. 
We find it useful to parametrise different momenta in the BF as displayed below

$$
\begin{aligned}
p & =m \bar{\gamma}(1,0,0, \bar{\beta}), \\
p^{\prime} & =m \bar{\gamma}(1,0,0,-\bar{\beta}), \\
u & =\gamma_{u}\left(1, \beta_{u} \sin \chi, 0, \beta_{u} \cos \chi\right), \\
q^{\prime} & =2 m \bar{\gamma}(1,0,0,0) .
\end{aligned}
$$

Note that the BF is the rest frame of $q^{\prime}$. The parametrisations in the HBF can be obtained by boosting these so as to obtain $u=(1,0,0,0)$.

The quantities $\bar{\gamma}, \gamma_{u}$ and $\chi$ are Lorentz invariant. It is easy to see that

$$
\bar{\beta}=\sqrt{\frac{\Psi-1}{\Psi+1}}, \quad \text { and } \quad \bar{\gamma}=\sqrt{\frac{\Psi+1}{2}} .
$$

As a result, the hard-scattering limit corresponds to taking $\bar{\gamma} \rightarrow \infty$. In addition, we can write an explicitly Lorentz-invariant form for $\gamma_{u}$,

$$
\gamma_{u}=\frac{u \cdot\left(p+p^{\prime}\right)}{\sqrt{\left(p+p^{\prime}\right)^{2}}}
$$

An explicitly invariant form of $\chi$ can also be written down.

\section{The Integrals}

In this appendix we investigate integrals of the form

$$
I=\int \frac{d^{4} k}{(2 \pi)^{4}} 2 \pi \delta\left(k^{2}\right) \Theta\left(k_{0}\right) \Theta\left(\eta q^{2}-q^{\prime} \cdot k\right) 2 B(k \cdot u ; T) F(k),
$$

where $B$ is the Bose distribution function and $F(k)$ is a scalar involving (possibly) $p, p^{\prime}$ and $u$. The second $\Theta$-function on the right restricts the phase space to soft modes. Since we are interested in the region $\eta \ll 1$, we shall freely use the hard scattering kinematics of Appendix B.

In the $\mathrm{BF}$ this phase space constraint takes the particularly simple form

$$
\Theta\left(\eta q^{\prime 2}-q^{\prime} \cdot k\right)=\Theta\left(2 m \eta \bar{\gamma}-k_{0}\right)
$$


The only constraint is on the values of $k_{0}$. For $T=0$, the Bose distribution drops out, and the only possible angular dependences are in $F(k)$. Hence, all the relevant integrals are best done in the $\mathrm{BF}$.

For $T>0$, the Bose distribution picks up angular factors in the BF. Hence, it is simpler to perform these integrals in the HBF. However, the constraint now cuts off part of the angular region in phase space. Fortunately, the analysis of this problem is not complicated. The constraint can be written as

$$
\Theta\left(\eta q^{\prime 2}-q^{\prime} \cdot k\right)=\Theta\left(\frac{2 m \eta \bar{\gamma}}{\gamma_{u}}-k_{0}\left(1-\beta_{u} \hat{\mathbf{q}}^{\prime} \cdot \hat{\mathbf{k}}\right)\right)
$$

where boldfaced symbols with carets denote unit 3-vectors.

The quantity within brackets on the right hand side lies between $1-\left|\beta_{u}\right|$ and $1+\left|\beta_{u}\right|$. As a result, the constraint picks a non-trivial angular region of integration only for $k_{-} \leq k_{0} \leq k_{+}$, where

$$
k_{-}=2 m \eta \bar{\gamma} \sqrt{\frac{1-\left|\beta_{u}\right|}{1+\left|\beta_{u}\right|}}, \quad k_{+}=2 m \eta \bar{\gamma} \sqrt{\frac{1+\left|\beta_{u}\right|}{1-\left|\beta_{u}\right|}} .
$$

For $k \leq k_{-}$, the full angular region is allowed, and for $k \geq k_{+}$, no phase space volume is allowed.

Now, the integral $I$ can be split up into an integration over $0 \leq k_{0} \leq k_{-}$ $\left(I_{1}\right)$ and another over $k_{-} \leq k_{0} \leq k_{+}\left(I_{2}\right)$. The angular integral in the first part is over the full sphere $\hat{\mathbf{k}}$, whereas that in the second part is restricted by the constraint.

This second integral becomes

$$
I_{2}=\frac{1}{8 \pi^{3}} \int_{k_{-}}^{k_{+}} k d k B(k ; T) f(k),
$$

where $f(k)$ is obtained from $F(k)$ by the appropriate angular integration. Since $B(k ; T)$ is monotonically decreasing, we have the bound

$$
\left|I_{2}\right| \leq \frac{B\left(k_{-} ; T\right)}{8 \pi^{3}} \int_{k_{-}}^{k_{+}} k d k|f(k)| .
$$

For all the integrands we are interested in, the growth with $\bar{\gamma}$ of the integral on the right hand side is polynomially bounded. Since $B\left(k_{-} ; T\right)$ decreases exponentially with $\bar{\gamma}, I_{2}$ is exponentially small in the hard scattering limit. As a result, it can be neglected in comparison with $I_{1}$. 
The angular integrals in the first part are unconstrained. Hence we find it useful to define the integrals

$$
\Omega_{i}=\int d \Omega F_{i}(k), \quad \text { where } \quad d \Omega=\frac{d \cos \theta d \phi}{4 \pi},
$$

with the convention that $F_{i}(k)$ is rendered dimensionless by dividing each factor of $p$ or $p^{\prime}$ by $m$ and $k$ by $k_{0}$. In the full computation we need many forms of $F_{i}(k)$. These, and the resulting angular integrals are listed in Table 1 .

For $Q \rightarrow \infty$, we can now write

$$
I \approx \frac{\Omega_{i}}{2 \pi^{2}} \int_{0}^{k_{-}} k d k B(k ; T) F(k, \theta, \phi) .
$$

In actual applications, we shall choose $k_{-}$to be large enough that

$$
\int_{0}^{k_{-}} d k k B(k ; T) \approx \frac{\pi^{2} T^{2}}{6} .
$$

A numerical evaluation of the incomplete Bose integral on the left shows that this approximation is valid to an accuracy of one part in thousand for $k_{-} \approx 8.6 T$. Now if we were to have $T \ll Q$, then we must either take $\eta \ll 1$ or $1-\left|\beta_{u}\right| \ll 1$ in order to satisfy this condition. Since $\eta$ is some fixed number, for $Q / T \gg 1$, it is possible to satisfy the constraint even for $\gamma_{u} \gg 1$.

In short, in the hard-scattering limit, we can approximate all integrals of the form in eq. (C.1) with exponential accuracy to obtain

$$
I_{i}=\frac{T^{2} \Omega_{i}}{12} .
$$

The angular integrals $\Omega_{i}$ are frame dependent, and are listed in Table 1 .

\section{Tensor Decompositions}

The cumulants are symmetric Lorentz tensors which can be written as an integral $I_{\mu_{1} \mu_{2} \cdots \mu_{n}}$ over a vector $k$, containing some vectors $V_{1}, V_{2}$, etc, in the integrand. As a result, they admit expansions of the form-

$$
I_{\mu_{1} \mu_{2} \cdots \mu_{n}}=\sum_{i} F_{i} Q_{\mu_{1} \mu_{2} \cdots \mu_{n}}^{i}
$$




\begin{tabular}{|c|c|c|c|}
\hline Integral & $F_{i}$ & HBF & BF \\
\hline$\Omega_{0}$ & 1 & 1 & 1 \\
\hline$\Omega_{1}$ & {$\left[m k_{0} / p \cdot k\right]$} & $L(\beta) / \gamma$ & $L(\bar{\beta}) / \bar{\gamma}$ \\
\hline$\Omega_{2}$ & {$\left[m^{2} k_{0}^{2} /(p \cdot k)^{2}\right]$} & 1 & \\
\hline$\Omega_{3}$ & {$\left[m^{2} k_{0}^{2} / p \cdot k p^{\prime} \cdot k\right]$} & $L\left(\beta_{\Psi}\right) / \Psi$ & \\
\hline$\Omega_{4}$ & {$\left[p^{\prime} \cdot k / p \cdot k\right]$} & {$[\phi+\Phi L(\beta) / \gamma] /(\beta \gamma)^{2}$} & $2 L(\bar{\beta})-1$ \\
\hline & & {$[\xi+\lambda L(\beta)] /(\beta \gamma)^{4}$,} & \\
$\Omega_{5}$ & {$\left[p^{\prime} \cdot k / p \cdot k\right]^{2}$} & $\xi=\Phi^{2}+2 \phi^{2}-\Gamma^{2}$, & $4 \bar{\gamma}^{2}+1-4 L(\bar{\beta})$ \\
& & $\lambda=\Gamma^{2}-\phi(\phi-2 \Phi / \gamma)$. & \\
\hline$\Omega_{6}$ & $m k_{0}\left[p^{\prime} \cdot k /(p \cdot k)^{2}\right]$ & {$[\Phi+\phi L(\beta) / \gamma] /(\beta \gamma)^{2}$} & $2 \bar{\gamma}-L(\bar{\beta}) / \bar{\gamma}$ \\
\hline
\end{tabular}

Table 1: Definitions and values of the angular integrals in the HBF and BF. The integration is over the full sphere $\hat{\mathbf{k}}$.We have used the mnemonics (a) $L(x)=\log ((1+x) /(1-x)) / 2 x$, (b) $\beta_{\Psi}=\sqrt{1-1 / \Psi^{2}}$, (c) $\phi=\gamma \gamma^{\prime}-\Psi$, (d) $\Phi=$ $\Psi \gamma-\gamma^{\prime}$ (e) $\Phi^{\prime}=\Psi \gamma^{\prime}-\gamma$ and (f) $\Gamma=\beta \beta^{\prime} \gamma \gamma^{\prime}$. $\Omega_{i}^{\prime}$ are defined by interchanging $p$ and $p^{\prime}$. In the HBF, their values are obtained by interchanging primed and unprimed quantities. In the $\mathrm{BF}, \Omega_{i}=\Omega_{i}^{\prime}$, since each integral is symmetric under $\bar{\beta} \rightarrow-\bar{\beta}$. 
The set of basis tensors $Q^{i}$ consists of all possible symmetric rank-n tensors which may be written using the vectors $V_{1}, V_{2}$, etc, and the metric tensor $g_{\mu \nu}$. We choose the basis tensors to be dimensionless.

The coefficients $F_{i}$ are Lorentz scalars, and given by the solution of the set of linear equations

$$
\mathcal{I}_{i}=\sum_{i} \mathbf{Q}_{i j} F_{j}, \quad \text { where } \quad \mathcal{I}_{i}=Q_{\mu_{1} \cdots \mu_{n}}^{i} I^{\mu_{1} \cdots \mu_{n}}, \quad \mathbf{Q}_{i j}=Q_{\mu_{1} \cdots \mu_{n}}^{i} Q^{j \mu_{1} \cdots \mu_{n}} .
$$

The matrix of contractions of the basis tensors, $\mathbf{Q}$, is symmetric. The integrals $\mathcal{I}_{i}$ are Lorentz invariant.

The only vectors involved in the problem at $T=0$ are $p$ and $p^{\prime}$. The tensors necessary for the mean momentum are then $p / m$ and $p^{\prime} / m$. For the variance, we need to consider the four tensors

$$
\begin{aligned}
& \mathcal{Z}_{\mu \nu}^{1}=g_{\mu \nu}, \quad \mathcal{Z}_{\mu \nu}^{2}=\frac{p_{\mu} p_{\nu}}{m^{2}}, \\
& \mathcal{Z}_{\mu \nu}^{3}=\frac{p_{\mu}^{\prime} p_{\nu}^{\prime}}{m^{2}}, \quad \mathcal{Z}_{\mu \nu}^{4}=\frac{p_{\mu} p_{\nu}^{\prime}+p_{\nu} p_{\mu}^{\prime}}{2 m^{2} \Psi} .
\end{aligned}
$$

The corresponding matrix of contractions is

$$
\mathbf{Z}=\left(\begin{array}{cccc}
4 & 1 & 1 & 1 \\
1 & 1 & \Psi^{2} & 1 \\
1 & \Psi^{2} & 1 & 1 \\
1 & 1 & 1 & \frac{1+\Psi^{2}}{2 \Psi^{2}}
\end{array}\right)
$$

The linear system to be solved is $\mathbf{Z} F=\mathcal{I}$.

For the covariance at $T>0$, the basis tensors must be built out of three vectors, $p, p^{\prime}$ and $u$. It is more convenient to work in terms of $u$ and the two vectors $\tilde{p}=p-(p \cdot u) u$ and $\tilde{p}^{\prime}=p^{\prime}-\left(p^{\prime} \cdot u\right) u$. These two vectors are orthogonal to $u$ [10. There are seven basis tensors, and they can be chosen to be

$$
\begin{array}{lrl}
\mathcal{T}_{\mu \nu}^{1} & =g_{\mu \nu}-u_{\mu} u_{\nu} & \mathcal{T}_{\mu \nu}^{2}=\frac{\tilde{p}_{\mu} \tilde{p}_{\nu}}{p^{2}} \\
\mathcal{T}_{\mu \nu}^{3}=\frac{\tilde{p}_{\mu}^{\prime} \tilde{p}_{\nu}^{\prime}}{p^{\prime 2}} & \mathcal{T}_{\mu \nu}^{4}=\frac{\tilde{p}_{\mu} \tilde{p}_{\nu}^{\prime}+\tilde{p}_{\nu} \tilde{p}_{\mu}^{\prime}}{2 p \cdot p^{\prime}} \\
\mathcal{T}_{\mu \nu}^{5}=u_{\mu} u_{\nu} & \mathcal{T}_{\mu \nu}^{6}=\frac{u_{\mu} \tilde{p}_{\nu}+u_{\nu} \tilde{p}_{\mu}}{2 u \cdot p} \\
\mathcal{T}_{\mu \nu}^{7}=\frac{u_{\mu} \tilde{p}_{\nu}^{\prime}+u_{\nu} \tilde{p}_{\mu}^{\prime}}{2 u \cdot p^{\prime}} &
\end{array}
$$


With this choice of tensors, the matrix of contractions is

$$
\mathbf{T}=\left(\begin{array}{ccccccc}
3 & -\gamma^{2} \beta^{2} & -\gamma^{\prime 2} \beta^{\prime 2} & -\frac{\phi}{\Psi} & 0 & 0 & 0 \\
-\gamma^{2} \beta^{2} & \gamma^{4} \beta^{4} & \phi^{2} & \frac{\gamma^{2} \beta^{2} \phi}{\Psi} & 0 & 0 & 0 \\
-\gamma^{\prime 2} \beta^{\prime 2} & \phi^{2} & \gamma^{\prime 4} \beta^{\prime 4} & \frac{\gamma^{\prime 2} \beta^{\prime 2} \phi}{\Psi} & 0 & 0 & 0 \\
-\frac{\phi}{\Psi} & \frac{\gamma^{2} \beta^{2} \phi}{\Psi} & \frac{\gamma^{\prime 2} \beta^{\prime 2} \phi}{\Psi} & \frac{\Gamma^{2}+\phi^{2}}{2 \Psi^{2}} & 0 & 0 & 0 \\
0 & 0 & 0 & 0 & 1 & 0 & 0 \\
0 & 0 & 0 & 0 & 0 & -\frac{\beta^{2}}{2} & -\frac{\phi}{2 \gamma \gamma^{\prime}} \\
0 & 0 & 0 & 0 & 0 & -\frac{\phi}{2 \gamma \gamma^{\prime}} & -\frac{\beta^{\prime 2}}{2}
\end{array}\right)
$$

We have used the notation for $\phi$ and $\Gamma$ as given in the caption of Table 1 .

For comparison with $T=0$, it is more convenient to express the results in terms of the tensor set

$$
\begin{aligned}
S_{\mu \nu}^{i}=\mathcal{Z}_{\mu \nu}^{i} \quad \text { for } i=1, \cdots 4, \quad S_{\mu \nu}^{5}=u_{\mu} u_{\nu}, \\
S_{\mu \nu}^{6}=\frac{u_{\mu} p_{\nu}+u_{\nu} p_{\mu}}{2 u \cdot p}, \quad S_{\mu \nu}^{7}=\frac{u_{\mu} p_{\nu}^{\prime}+u_{\nu} p_{\mu}^{\prime}}{2 u \cdot p^{\prime}} .
\end{aligned}
$$

These can be expanded in terms of the set $\mathcal{T}^{i}$. We have $T_{\mu \nu}^{i}=\mathbf{S}^{i j} S_{\mu \nu}^{j}$, where

$$
\mathbf{S}=\left(\begin{array}{ccccccc}
1 & 0 & 0 & 0 & -1 & 0 & 0 \\
0 & 1 & 0 & 0 & \gamma^{2} & -2 \gamma^{2} & 0 \\
0 & 0 & 1 & 0 & \gamma^{\prime 2} & 0 & -2 \gamma^{\prime 2} \\
0 & 0 & 0 & 1 & \frac{\gamma \gamma^{\prime}}{\Psi} & -\frac{\gamma \gamma^{\prime}}{\Psi} & -\frac{\gamma \gamma^{\prime}}{\Psi} \\
0 & 0 & 0 & 0 & 1 & 0 & 0 \\
0 & 0 & 0 & 0 & -1 & 1 & 0 \\
0 & 0 & 0 & 0 & -1 & 0 & 1
\end{array}\right) .
$$

\section{References}

[1] M. Gyulassy and M. Plümer, Nucl. Phys. B 346 (1990) 1;

M. Gyulassy and X.-N. Wang, Nucl. Phys. B 420 (1994) 583;

J.-C. Pan and C. Gale, Phys. Rev., D 50 (1994) 3235. 
[2] S. Gupta, Phys. Lett., B 347 (1995) 381.

[3] F. Bloch and A. Nordsieck, Phys. Rev., 52 (1937) 54;

See also D. R. Yennie, S. C. Frautschi and H. Suura, Ann. Phys., 13 (1961) 379, and references therein.

[4] T. D. Lee and M. Nauenberg, Phys. Rev., 133 (1963) 1549. T. Kinoshita, J. Math. Phys., 3 (1962) 650.

[5] T. Grandou, M. LeBellac and D. Poizat, Phys. Lett., B 249 (1990) 478 and Nucl. Phys., B 358 (1991) 408;

T. Altherr, Phys. Lett., B 262 (1991) 314.

[6] T. Altherr, P. Aurenche and T. Becherrawy, Nucl. Phys., B 315 (1989) 436.

[7] P. V. Landshoff and M. Taylor, Nucl. Phys., B 430 (1994) 683.

[8] H. A. Weldon, Phys. Rev., D 49 (1994) 1579.

[9] N. P. Landsman and Ch. G. van Weert, Phys. Rep., 145 (1987) 141;

A. Niemi and G. Semenoff, Nucl. Phys., B 230 (1984) 181.

[10] H. A. Weldon, Phys. Rev., D 26 (1982) 1394.

[11] I. Hinchliffe and S. F. Novaes, Phys. Rev., D 38 (1988) 3475;

R. Kauffman, Phys. Rev., D 44 (1991) 1415;

E. L. Berger, Rui-ben Meng, Phys. Rev., D 49 (1994) 3248.

[12] J. M. Jauch and F. Rohrlich, Theory of Photons and Electrons, p. 390, Addison-Wesley, Cambridge, 1955.

[13] E. Braaten and R. Pisarski, Nucl. Phys., B 337 (1990) 569.

[14] S. D. Ellis, N. Fleishon and W. J. Stirling, Phys. Rev., D 24 (1981) 1386. 